\title{
BMJ Open Study protocol for the evaluation of long-term effects of the school-based obesity prevention program Lekker Fit! ('enjoy being fit'): a retrospective, controlled design
}

\author{
Michel Sebastiaan Smit (D) , ${ }^{1}$ Hein Raat (D) , ${ }^{1}$ Famke Mölenberg (D) , ${ }^{1}$ \\ Mireille Eleonore Gabriëlle Wolfers, ${ }^{2}$ Rienke Bannink, ${ }^{3}$ Wilma Jansen (D) ${ }^{1,2}$
}

To cite: Smit MS, Raat $\mathrm{H}$, Mölenberg $\mathrm{F}$, et al. Study protocol for the evaluation of long-term effects of the school-based obesity prevention program Lekker Fit! ('enjoy being fit'): a retrospective, controlled design. BMJ Open 2021;11:e046940. doi:10.1136/ bmjopen-2020-046940

- Prepublication history for this paper is available online. To view these files, please visit the journal online (http://dx.doi. org/10.1136/bmjopen-2020046940).

Received 17 November 2020 Accepted 30 July 2021

A) Check for updates

(c) Author(s) (or their employer(s)) 2021. Re-use permitted under CC BY-NC. No commercial re-use. See rights and permissions. Published by BMJ.

${ }^{1}$ Public Health, Erasmus MC, Rotterdam, The Netherlands ${ }^{2}$ Social Development, Gemeente Rotterdam, Rotterdam, The Netherlands

${ }^{3}$ Policy and Research, CJG Rijnmond, Rotterdam, The Netherlands

Correspondence to

Dr Wilma Jansen;

w.jansen@Rotterdam.nl

\section{ABSTRACT}

Introduction Preventive interventions to reduce overweight and obesity in childhood and adolescence are studied on their effectiveness worldwide. A number with positive results. However, long-term effects of these interventions and their potentially wider influence on wellbeing and health have been less studied. This study aims to evaluate the long-term effects of a multicomponent intervention in elementary school children targeting individual behaviour as well as environment (Lekker Fit!). The primary outcomeis body mass index and the secondary outcomes are waist circumference, weight status, physical fitness, lifestyle, psychosocial health and academic performance.

Methods and analysis In a naturalistic effect evaluation with a retrospective, controlled design adolescents in secondary schools, from intervention and non-intervention elementary schools, will be compared on a wide set of outcome variables. Data will be collected by questionnaires and through anthropometric and fitness measurements by trained physical education teachers and research assistants. Baseline data consist of measurements from the adolescents at the age of 5 years old and are gathered from preventive youth healthcare records, from before the intervention took place. Multilevel regression models will be used and adjusted for baseline measurements and potential confounding variables on the individual and environmental level. Furthermore, propensity scores will be applied.

Ethics and dissemination The study has been approved by the Medical Research Ethics Committee of the Erasmus Medical Centre, Rotterdam, The Netherlands (permission ID: MEC-2020-0644). Study findings will be disseminated in peer-reviewed journals and by conference presentations.

Trial registration number NL8799. Pre-results.

\section{INTRODUCTION}

The prevalence of overweight and obesity in children has been a growing health concern for many years worldwide. ${ }^{1-3}$ Recent estimation from the WHO European Childhood
Strengths and limitations of this study

- Studies regarding the sustainability of effects of school-based obesity prevention programmes are needed.

- Effects on a broad set of outcome variables will be assessed.

- A controlled study design will be used with retrospective collection of baseline data and adjustment for the non-randomised design by a range of confounders.

- Study population will be diverse in age, socioeconomic background, ethnic background, grade and school level.

Obesity Surveillance Initiative (COSI) indicates an overweight prevalence of 9\%-43\% for boys and $5 \%-43 \%$ for girls, with numbers varying across countries. ${ }^{45}$ Furthermore, an obesity prevalence of $2 \%-21 \%$ for boys and $1 \%-19 \%$ for girls was reported by the COSI. ${ }^{45}$ Although a recent stabilisation in the trend of the overweight and obesity prevalence in children has been demonstrated in highincome countries, the overall prevalence of overweight in childhood remains high. ${ }^{2}{ }^{6}$ In 2019 in the Netherlands, $12.0 \%$ of the children (aged 4-12 years old) were overweight, including $2.0 \%$ obese children. ${ }^{7}$ For adolescents (aged 12-16 years old), overweight prevalence was $14.7 \%$ of the population, including $1.9 \%$ obese adolescents. ${ }^{7}$

Childhood overweight and obesity have been associated with numerous adverse health consequences, like cardiovascular disorders, type 2 diabetes and psychosocial health problems. ${ }^{8-13}$ Furthermore, children with overweight or obesity are at higher risk for obesity in adulthood than their normal weight peers. ${ }^{14}{ }^{15}$ In addition, individuals with 
obesity pose a substantial financial burden on the global healthcare systems as their individual medical costs are $30 \%$ higher. $^{16}$ The combination of the economic consequences, health consequences and high prevalence warrants the implementation of effective interventions to prevent and reduce overweight and obesity in children. ${ }^{617}$

Physical acitivity and diet, being factors associated with the development of overweight and obesity, ${ }^{18}{ }^{19}$ often form important entry points for those interventions. A recent Cochrane review, addressing interventions for the prevention of obesity, reported that combined diet and physical activity interventions do reduce body mass index (BMI) z-scores for children aged 6-12 years old, although the level of evidence is low. ${ }^{20}$ Schools have been suggested to be the optimal place to deliver the interventions due to continuous contact with children, ${ }^{21}$ and reaching children with a wide range of different backgrounds. ${ }^{22}$ Two recent reviews provide evidence that school-based interventions are generally effective in the reduction of children's weight gain. ${ }^{1723}$ However, little is known about the sustainability of effects by obesity prevention interventions as long-term studies are scarce. ${ }^{24}{ }^{25}$ The authors of the Cochrane review indeed suggest that interventions and strategies to prevent obesity in children should include follow-up measurements over several years. ${ }^{20}$ Furthermore, the wider effects of school-based interventions including a physical acitivity component on health and well-being have been less studied, although the association between physical activity and both academic performance $^{2627}$ and psychosocial health and well-being ${ }^{28-30}$ in children and adolescents have been well documented.

The multicomponent obesity prevention programme Lekker Fit! (translated as "enjoy being fit ${ }^{31}$ ) for schoolaged children was developed and implemented in 2005 via elementary schools in Rotterdam, the Netherlands, targeting children aged 6-12 years old. The results of a randomised controlled trial (RCT) performed in 2006-2007 demonstrated positive intervention effects on overweight prevalence, waist circumference and aerobic fitness among elementary school children in grades $3-5$ after 1 year of intervention. ${ }^{32}$ The long-term effects of Lekker Fit! (after leaving elementary school) as well as the wider effects on psychosocial health and academic performance have not been studied yet. This knowledge is important to determine whether beneficial effects of Lekker Fit! sustain into adolescence and whether wider effects are present. To the authors' knowledge, no studies are available for the assessment of the long-term effectiveness of multicomponent school-based interventions on a set of outcome variables including weight status, fitness, lifestyle, psychosocial health and academic performance.

Therefore, the main objective of this study is to evaluate the long-term effects of Lekker Fit! on (a) the primary outcome BMI, and (b) the secondary outcomes waist circumference, weight status, physical fitness, lifestyle and lifestyle determinants, psychosocial health and academic performance. In subgroup analysis, we will explore if any encountered effects are different for gender, socioeconomic status and time since intervention. Moreover, dose-effect assocations will be explored if the data provide sufficient variability in the amount of intervention years. Finally, on an exploratory basis, we will investigate the participants' appreciation of the Lekker Fit! intervention and similar components from regular school programmes.

Our hypothesis is that adolescents who have attended a Lekker Fit! elementary school exhibit healthier scores than their peers who have attended a regular elementary school on the set of primary and secondary outcome variables. However, we do not know the relationship between longer follow-up and the intervention effects, due to the scarce literature on the sustainability of such intervention effects into adolescence. Furthermore, we hypothesise that more years of Lekker Fit! intervention on an elementary school leads to better scores on the set of outcome variables, due to the prolonged exposure to this behavioural changing intervention.

\section{METHODS AND ANALYSIS}

\section{Lekker Fit! intervention and regular school program}

In 2005, to halt the rise in obesity among children, the City of Rotterdam, the Netherlands has developed and implemented the intervention programme Lekker Fit! (translated as 'enjoy being fit'). ${ }^{31}$ This programme has been implemented in collaboration with elementary schools in Rotterdam targeting children aged 6-12 years old and at a later stage with day care organisations. In the Dutch school system, children attend elementary school for 8 years (4-12 years old) and start secondary school at age 12. In 2020, about half of all elementary schools (94) in Rotterdam have adopted Lekker Fit! into their education programme. ${ }^{33}$

Although the Lekker Fit! programme has no direct focus on reducing overweight, the contribution of the programme to reducing overweight is expected to be the consequence of a healthy diet and active lifestyle. The intervention entails multiple components (table 1). In comparison with regular school programmes, these include an additional third physical education (PE) lesson per week, professional PE teachers instead of regular classroom teachers providing the PE lessons, voluntary additional physical activities outside school hours, the promotion of drinking water and the promotion of a healthy diet and lifestyle. ${ }^{33}$

Besides targeting individual behaviours of children, Lekker Fit! targets the obesogenic environment of the children and involves parental engagement. ${ }^{31} 32$ The strategy for behavioural change is mainly based on the theory of planned behaviour (TPB), ${ }^{31}$ while several components of Lekker Fit! are based on specific theories such as social marketing. ${ }^{34}$ The TPB states that a behaviour is being influenced by the intention towards that behaviour. The intention itself is being influenced by the individual's attitude, social norm and self-efficacy. ${ }^{35}{ }^{36}$ Lekker Fit! primarily targets children in the socioeconomically 
Table 1 Components (and their year of introduction) of the Lekker Fit! intervention on elementary schools in comparison with the regular elementary school programme

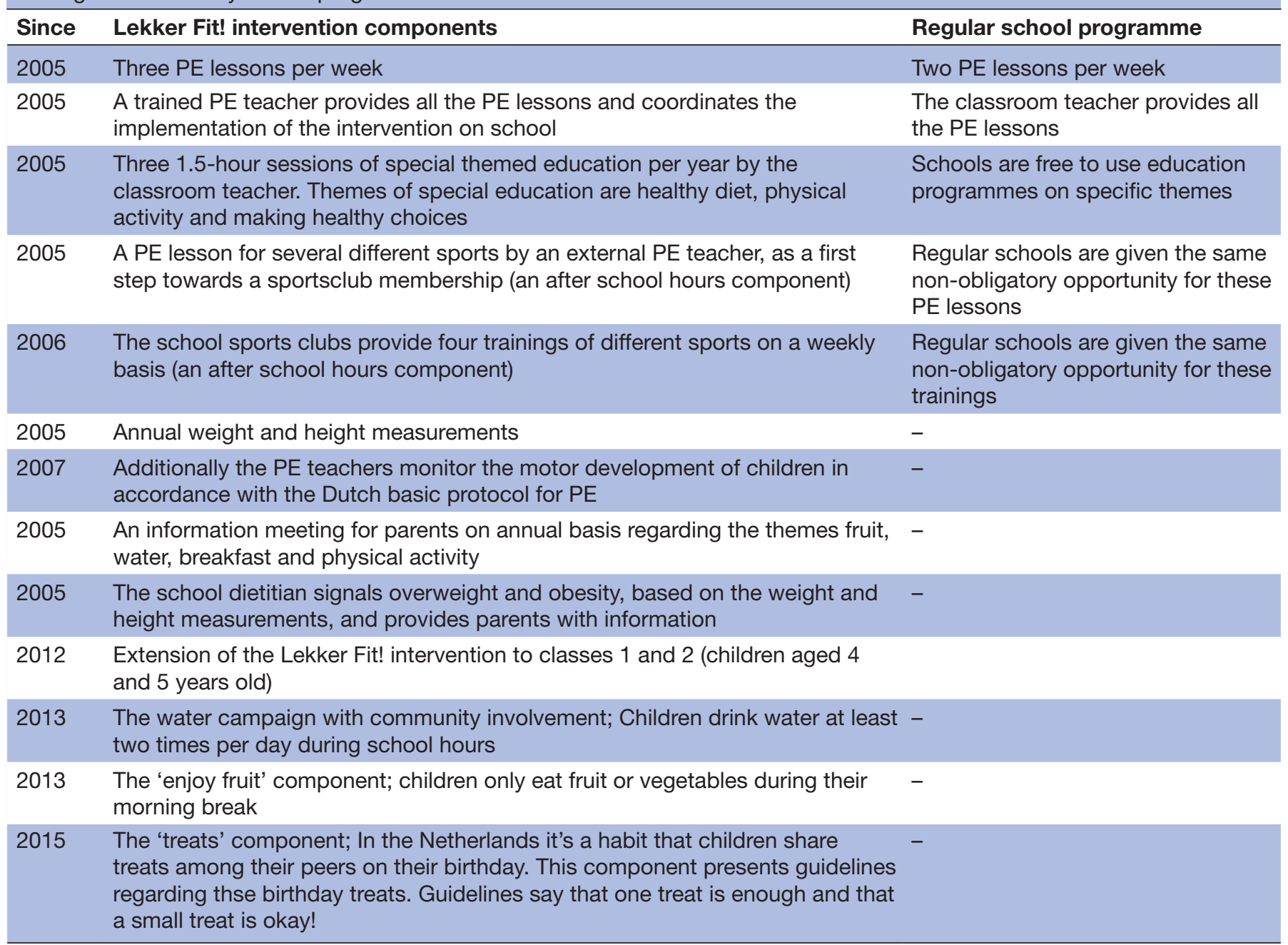

PE, physical education.

disadvantaged neighbourhoods, because of a higher prevalence of overweight and obesity and therefore a higher risk at metabolic diseases in those neighbourhoods. ${ }^{3738}$

\section{Study design}

In order to determine the long-term effects of the Lekker Fit! intervention, a naturalistic effect evaluation ${ }^{39-41}$ with a retrospective, controlled design will be conducted

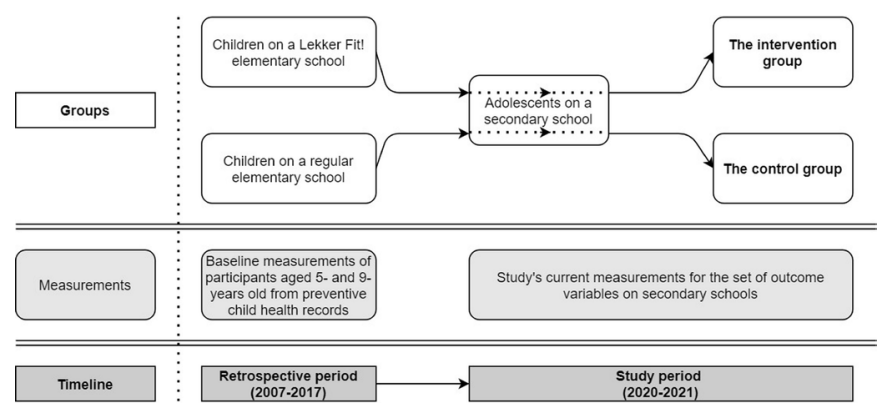

Figure 1 The naturalistic effect evaluation with a retrospective, controlled design of this study. (figure 1). We will recruit adolescents aged 12-18 years old on secondary schools for participation in the study. This allows for a follow-up period of up to 6 years. Due to the COVID-19 pandemic, a prolonged recruitment and data collection period is applied in this study. The recruitment period ranges from September 2020 up to and including September 2021. The data collection period continues until December 2021.

We will compare the adolescents who attended Lekker Fit! elementary schools-the intervention groupwith adolescents who attended regular non-Lekker Fit! elementary schools-the control group. Given the naturalistic non-randomised design, correction for confounding variables will be applied. Correction for available pre-intervention baseline measurements around the age of 5 years old will be applied by obtaining data from the regional preventive youth healthcare provider (CJG Rijnmond). Furthermore, available measurements at the age of 9 years old will be obtained to serve as an additional control measurement. Thus, the set of baseline 
data ranges from the period 2007-2017, depending on the current ages of the participating adolescents. Confounding variables and baseline measurements will be described in detail below. We used the Standard Protocol Items: Recommendations for Interventional Trials checklist (online supplemental file 1) to address all recommend items in our study protocol. ${ }^{42}$

\section{Study population and recruitment}

We will include 2218 adolescents aged $12-18$ years in this study (see the Power considerations section). We expect to include 20-25 secondary schools in Rotterdam, from neighbourhoods with divergent socioeconomic backgrounds, in this study. They will receive an informative letter to explain the nature, relevance, objectives and measurements of this study. Only after a school has given consent for its participation, will the adolescents in that school be approached for participation in close collaboration with the school. We will recruit adolescents from all different school levels and grades within the age range. The targeted adolescents and their parents will receive an information letter. Additionally, all relevant information about the study will be available on a website (URL: https://www.rotterdam.nl/onderzoeklekkerfit). Comprehensive information will be given about all the relevant topics regarding the study and the consequences of participation. Adolescents are asked to give digital informed consent before they are eligible for participation in this study. For the adolescents who are under the age of 16 years old, digital informed consent by one parent will also be obtained in accordance with Dutch legislation.

\section{Lekker fit! intervention group and control group}

For all adolescents in this study, their school career (specified per school year) will be obtained from the municipal records in Rotterdam. Specific permission for the collection and coupling of this data will be asked within the digital informed consent forms. Combined with information from the City of Rotterdam about which elementary schools implemented Lekker Fit!, we are able to assign the adolescents to the intervention group or control group. Adolescents who attended elementary schools outside Rotterdam were not exposed to Lekker Fit! and will be assigned to the control group. Children may move between schools and change between the intervention condition and the control condition. Based on the accurate year by year school career data from the municipality, we will be able to determine exposure to Lekker Fit! in years and years since intervention for all adolescents.

\section{New data collection}

Participants are asked to complete a digital questionnaire using LimeSurvey software during school hours and to engage in physical measurements during their PE class. LimeSurvey will also be used by the researchers to manage the digital consent forms of participants and parents.
Furthermore, we will collect information about possible confounding variables that might have an influence on the effect of the Lekker Fit! intervention. In this respect, measures for age, gender, ethnic background, household situation and socioeconomic background will be obtained from participants and environmental measures like the presence of a healthy school canteen and the presence of any additional psychosocial or physical interventions on the secondary schools will be collected. All the instruments that will be used for data collection are described within the Measurements section.

\section{Retrospective data collection}

Retrospective baseline data will be retrieved from the CJG Rijnmond archives. The CJG Rijnmond is a regional preventive youth healthcare provider for children in the larger Rotterdam area. The CJG Rijnmond invites all children for growth and health monitoring during childhood. Collected baseline data will involve measures of anthropometrics, BMI and psychosocial health data. The data consist of measurements from the participants around the age of 5 years old and 9 years old (figure 1). Baseline data around the age of 5 years old will provide information about participants before the Lekker Fit! intervention is entered (Lekker Fit! starts in grade 3 of elementary school). Baseline data around the age of 9 years old will be obtained as an additional control measurement. Furthermore, the child records consist of information about any additional professional guidance that participating adolescents received for being overweight during childhood.

\section{Measurements}

Primary and secondary outcome variables

The primary outcome variable in this study is BMI. The secondary outcome variables in this study are waist circumference, weight status, physical fitness, lifestyle and lifestyle determinants, psychosocial health and academic performance.

Trained PE teachers and research assistants will measure body weight, body length and waist circumference. Body weight will be measured to the nearest $0.1 \mathrm{~kg}$ and body length and waist circumference will be measured to the nearest $0.1 \mathrm{~cm}$. A fixed protocol will be used in which the adolescents will be measured (apart from their peers) with light clothing, without shoes. BMI will then be calculated and BMI-for-age z-scores (zBMI) will be determined based on international growth standards for school-aged children and adolescents. ${ }^{4344}$ Based on BMI-for-age z-scores, adolescents' weight status will be categorised as underweight $(\mathrm{zBMI}<-2)$, normal weight $(-2 \leq \mathrm{zBMI} \leq 1)$, overweight $(\mathrm{zBMI}>1)$ or obese $(\mathrm{zBMI}>2)$ using common cut-offs according to WHO standards. ${ }^{43} 45$ Physical fitness will be measured by the 20m-Shuttle Run test according to a standardised protocol under guidance of the PE teacher in which the outcome will be the number of stages completed. ${ }^{46}$ The $20 \mathrm{~m}$-Shuttle Run test 
is an acceptable, feasable and reliable method for determination of cardiorespiratory fitness in youth. ${ }^{47}$

The LimeSurvey questionnaire will be used to obtain information on the outcome variables lifestyle and lifestyle determinants, psychosocial health and academic performance. The Short Questionnaire to Assess Healthenhancing physical activity (SQUASH) will be used to assess adherence to physical activity guidelines. ${ }^{48}$ The SQUASH is being used by Dutch government agencies to monitor individuals concerning physical activity guidelines. ${ }^{49}$ The SQUASH was validated using the doubly labelled water method. The SQUASH was found to be a valid self-report tool for measuring physical activity energy expenditure for adolescents. ${ }^{49}$ Furthermore, participants will be asked if they currently have a membership at a sportclub and if they had a sportclub membership during elementary school.

Psychosocial health will be assessed by the Dutch version of the Strengths and Difficulties Questionnaire (SDQ) ${ }^{50}$ The SDQ contains 25 items which are evenly distributed over the domains of emotional symptoms, behavioural problems, hyperactivity/inattention, problems with peers and pro-social behaviour. The SDQ leads to a total score as well as five domain scores. Its Dutch version is classified as a valid and reliable instrument for the first identification of psychosocial problems in adolescents. ${ }^{51}$

Determinants of a healthy lifestyle are measured using items based on the TPB. ${ }^{35}$ Questions are included for healthy behaviours that are promoted by the Lekker Fit! intervention using the guidelines by Ajzen. ${ }^{52}$ The healthy behaviours, for which determinants will be measured, include ${ }^{5354}$ :

1. 1 hour of moderate intensely exercising every day.

2. Three times per week muscular and bone strengtening exercises.

3. Consciously making healthy choices in diet.

4. Two pieces of fruit every day.

5. $250 \mathrm{~g}$ of vegetables every day (approximately four serving spoons).

6. Limiting sugar-sweetened beverage consumption.
Participants are asked to rate their attitude, social norm, self efficacy, intention and actual behaviour (TPB items) towards these standards of healthy behaviour to derive the determinants for a healthy and active lifestyle (example illustrated in table 2).

Academic performance is measured by the question 'how well is your academic performance as judged by your teacher in comparison with the academic performance of your classmates?'. This item, derived from the International Health Behaviour in School-aged Children Study, was confirmed to be valid and useful to distinguish respondents who get good grades from respondents who do not get good grades. ${ }^{55}$ Furthermore, academic performance will be captured by the school level (preparatory vocational secondary education or senior general secondary education or university preparatory education) of the adolescent.

\section{Possible confounders}

Possible confounding variables that we will collect are participant's current age, gender, ethnic background, household situation, socioeconomic background and professional guidance for overweight during childhood.

Within the questionnaire, the adolescent's, mother's and father's country of birth will be asked. According to Statistics Netherlands, adolescents will be classified for ethnic background based on their mother's country of birth (to take into consideration the cultural background of the most frequent primary caregiver), unless it is the Netherlands. In that case, adolescents will be classified for ethnic background based on their father's country of birth (55).

Regarding household situation, we assess whether the participant lives alone, in a two parent or single parent household.

Socioeconomic background will be assessed by questions on parents' financial difficulties (Did you experience any financial burden in expenses in your household in the last 12 months?) and unemployment payments (Did one of your parents receive an unemployment

Table 2 Example of questions to asses the outcome measure determinants of a healthy and active lifestyle following the guidelines of Ajzen according to the theory of planned behaviour

\begin{tabular}{|c|c|c|}
\hline \multicolumn{3}{|c|}{ Healthy behaviour } \\
\hline \multicolumn{3}{|c|}{ Eating two pieces of fruit every day } \\
\hline TPB items & Question asked & Rating \\
\hline Perceived norm & $\begin{array}{l}\text { Most people who are important to me approve that I eat two } \\
\text { fruits every day for the next } 3 \text { months }\end{array}$ & Disagree 1/2/3/4/5/6/7 Agree \\
\hline Intention & I intend to eat two pieces of fruit every day for the next 3 months & Unlikely 1/2/3/4/5/6/7 Likely \\
\hline Past behaviour & In the past 3 months, I have eaten two pieces of fruit every day & False $1 / 2 / 3 / 4 / 5 / 6 / 7$ True \\
\hline
\end{tabular}

TPB, theory of planned behaviour. 
payment in the last 12 months?). Furthermore, socioeconomic neighbourhood indicators will be obtained for adolescents based on their postal code and/or elementary schools attended.

Other possible confounding variables that are embedded within the questionnaire include measurements for pubertal development. Pubertal development is measured with the Dutch version of the self-report Pubertal Development Scale. ${ }^{56}{ }^{57}$ These items are used to determine pubertal status of the participants and will be used to adjust weight status measures. For male participants, questions on pubertal development include growth spurt, body hair, facial hair, voice change and skin changes, whereas for female participants, questions include growth spurt, body hair, breast development, menstruation and skin changes.

On a school level, we will collect the following information from secondary schools:

1. The number of PE lessions per week.

2. The presence of dietetic programmes (for instance a healthy school canteen programme).

3. The presence of preventive mental health and well-being programme (for instance anti-bullying programmes).

\section{Appreciation of the intervention or similar components on regular} elementary schools

Within the questionnaire adolescents are asked to evaluate on several components of the Lekker Fit! intervention (or evaluate on similar components in regular elementary schools for the control group). Adolescents are asked to evaluate the quantity and quality of their PE lessons and quality of their PE teachers on elementary school. Furthermore, they are asked to rate their attendance and the quality regarding organised physical activites outside school hours. These items allow us to analyse the subjective experiences of participants during elementary school and allow us to compare appreciation of the Lekker Fit! intervention with the regular school programme.

\section{Power considerations}

A statistical a priori power analysis was performed for the estimation of sample size. ${ }^{58}$ With an alpha $=0.05$, power $=0.80$ and taking into account the cluster design, we need a sample size of $n=2218$ in total to find an absolute BMI difference of $0.3 \mathrm{~kg} / \mathrm{m}^{2}$ between the intervention and control group. ${ }^{58}$ Hereby assuming an SD of 3.0 , clustering within schools accounting for $4 \%$ of the variance (Intraclass correlation 0.04), a correlation of 0.75 for baseline and follow-up measurements. ${ }^{59}$ Further assuming a $30 \%$ participant loss to non-response, we aim to invite 3169 adolescents for participation in the study.

\section{Statistical analysis}

Descriptive statistics will be used to describe the characteristics of the participants in the total study population and separately for the intervention group and the control group. To study the effects of Lekker Fit!, we will use logistic and linear regression models to evaluate the longterm effects of the Lekker Fit! intervention for all primary and secondary outcome variables. Multilevel analyses will be used to correct for the clustering within secondary schools. The exposure to the Lekker Fit! intervention (intervention/control group) forms the independent variable. All baseline measurements and confounding variables will be added to the models as covariates. Interaction terms for intervention and the variables gender, ethnic background, educational level and time since intervention will be tested. Depending on results, exploratory subgroup analysis will be performed. Moreover, if the data provide sufficient variability in the amount of intervention years for participants, further exploratory analysis will be performed to evaluate possible dose-response associations (using years of intervention exposure). We anticipate that adolescents in the intervention group and control group may differ regarding several baseline characteristics. These baseline characteristics may have had an influence on the chance of receiving the Lekker Fit! intervention or not. Therefore, we will also perform propensity score adjusted comparisons of effects between intervention and control groups, by using propensity scores as a covariate in the model, to account for these possible bias due to the non-randomised design of this naturalistic study. The propensity score will be calculated using a model based on all baseline and confounding variables that will be used as covariates in the analysis on intervention effects. Multiple imputation techniques will be used to handle missing baseline and covariate data.

\section{Patient and public partnership}

No participants were involved in the creation and design of this study. A sample of the study population was first involved in this study by testing the questionnaires on quality and feasibility. Individual data may be disseminated to participants who are interested in their personal outcome variables. Participating secondary schools will receive data on school level, which can be used for their school policies. They will be encouraged to share the results with their students.

\section{DISCUSSION}

In this article the study protocol for a naturalistic effect evaluation with a retrospective, controlled design is described. The aim of this study is to evaluate the longterm effects of the Lekker Fit! intervention on (a) the primary outcome BMI and (b) the secondary outcomes waist circumference, weight status, physical fitness, lifestyle and lifestyle determinants, psychosocial health and academic performance.

Earlier studies have demonstrated a number of positive intervention effects for the Lekker Fit! intervention. ${ }^{32} 60$ An RCT on the effectiveness of Lekker Fit! was conducted a decade ago. ${ }^{32}$ Findings included positive intervention effects for weight status, waist circumference and physical fitness for 6-9years old children in elementary schools, 
although no positive effects on BMI were found. The Lekker Fit! intervention programme has been updated with several additional components since then (table 1); among others a water campaign component which incorporated multiple stakeholders in the neighbourhood of the school. The effectiveness of the water campaign was studied in a controlled design and provided evidence for the reduction of children's sugar-sweetened beverages consumption. ${ }^{60}$

Weihrauch-Blüher and colleagues recommended to combine behaviour-oriented intervention programmes, that were found to have only limited effects, with community-oriented components in order to reach sustainable effectiveness of obesity prevention interventions for children and adolescents. ${ }^{61}$ The Lekker Fit! intervention is such a multicomponent school-based intervention targeting the individual child and its environment. ${ }^{31-33}$ Lekker Fit! is thereby primarily targeting children in the socioeconomically disadvantaged neighbourhoods, because those children are at higher risk for the development of overweight and obesity. ${ }^{3738}$

The recent Cochrane review on obesity prevention interventions included 64 studies on school-based interventions, and 3 studies on school-based interventions with a community element for children aged 6-12 years old. ${ }^{20}$ They indicate that most of the evidence in their review is based on interventions of 12 months or less. They emphasise that research on long-term effects of completed studies would provide important information on the sustainability of behaviour change and impact on weight. ${ }^{20}$ Since long-term studies are scarce, little is known about the sustainability of effects ${ }^{24} 25$ or about the determinants that explain whether results are sustainable or not. Furthermore, little is known about the wider benefits that these interventions have on health and well-being of children and adolescents, although associations between physical activity and both academic performance ${ }^{2627}$ and psychosocial health and well-being ${ }^{28-30}$ in children and adolescents have been reported.

To the authors' knowledge, no studies are yet conducted for the measurement of the long-term effectiveness of multicomponent school-based interventions on the broad set of outcome variables including weight status, fitness, psychosocial health and academic performance. The Lekker Fit! intervention in elementary schools targets children up to 12 years old. As this study includes adolescents aged 12-18 years, long-term effects up to 6 years will be assessed. Additional subgroup analysis will provide insight in the interaction effects between intervention and follow-up length. The current study will therefore contribute to this field of scarce knowledge and expand the insights in the long-term effects of multicomponent school-based interventions and into the sustainability of intervention effects. Adding to this knowledge helps policymakers and intervention developers to decide on further implementation and intervention development.

RCTs are considered the gold standard for effectiveness evaluations of an intervention. ${ }^{62}$ Choosing for a randomised controlled design, with the length of the follow-up period we employ, would be almost impossible as it would be unethical to withhold schools from implementing an intervention programme that was already proven at least partly effective. The non-randomised design of our study can be regarded as the main limitation. We acknowledge that the assessment of the longterm effects of Lekker Fit! by a naturalistic evaluation design $^{39-41}$ is potentially subject to selection bias, which could arise by the fact that schools are not randomly chosen for implementation of the Lekker Fit! intervention. This limitation is accounted for by using propensity scores in the analysis ${ }^{63}$ to reduce selection bias. We also acknowledge there are factors that possibly influence our outcome measures besides the Lekker Fit! intervention on elementary school. Therefore, we will include preintervention baseline measurements and a broad spectrum of possible confounding variables on the individual and environmental level. The retrospective, controlled design with the application of propensity score analysis we choose seems best suited to overcome this limitation. Further, the self-report for physical activity can be regarded as a limitation. However, the SQUASH questionnaire was found to be a valid self-report tool for measuring physical activity energy expenditure for adolescents and is less costly than direct measurements. ${ }^{49}$ Self-report items may also manifest recall bias. To minimise recall bias for the items in our questionnaire, the items are easy to understand and sometimes provided with additional explanation or relatable examples.

On the other hand, we do not rely on self-report regarding anthropometric measurements or fitness. This can be regarded as a strength. Further, the naturalistic effect evaluation design of this study also allows us to observe subjects in 'a real world' setting instead of in a highly controlled experimental setting. A second strength of this practice-based design is therefore that it provides strong external validity of the results by providing a realistic representation of the 'practice-based' setting. ${ }^{39} 40$ This study hereby measures a broad set of outcome variables for a wide picture of the effects of Lekker Fit! on health in youth. The fact that we include a large sample size of participants with different socioeconomically backgrounds might be considered a strength of this study. It provides us with the opportunity to further generalise our findings to several populations characterised by apparent socioeconomic inequalities.

In conclusion. this paper describes the design of a study to determine the sustained effects of the schoolbased Lekker Fit! intervention, a multicomponent intervention targeting overweight and physical inactivity in children. This study will provide insight in the long-term intervention effects and will extend insights in a variety of outcome measures including BMI, waist circumference, weight status, physical fitness, lifestyle and lifestyle determinants, psychosocial health and academic performance. 


\section{ETHICS AND DISSEMINATION}

\section{Ethical statement}

The Medical Research Ethics Committee of the Erasmus Medical Centre, Rotterdam, The Netherlands decided that the regulations from the Dutch Medical Research Involving Human Subjects Act (Dutch abbreviation WMO) do not apply to this research protocol. Therefore permission was granted by the committee for the execution of this study and for publications in a later stage of the study (permission ID: MEC-2020-0644). The research proposal has been registered in the Dutch trial register NTR, in which effect-studies are registered that are conducted in the Netherlands.

\section{Dissemination statement}

The project team will disseminate the findings from this scientific study by conference presentations and scientific peer-reviewed journals.

Twitter Hein Raat @heinraat and Famke Mölenberg @FamkeMolenberg

Acknowledgements The authors would like to thank all the involved advisors for their role in the setup of this study.

Contributors MSS, HR, FM, MEGW, RB and WJ contributed to the design of the study and the development of the protocol for this study. MSS mainly wrote the manuscript and HR, FM, MEGW, RB and WJ contributed to the manuscript by critical revisions and giving comprehensive feedback on multiple drafts. MSS, HR, FM, MEGW, RB and WJ read and approved the final manuscript.

Funding This study is made possible by funding of the City of Rotterdam Sports Department.

Competing interests None declared.

Patient and public involvement Patients and/or the public were involved in the design, or conduct, or reporting, or dissemination plans of this research. Refer to the Methods section for further details.

Patient consent for publication Not required.

Provenance and peer review Not commissioned; externally peer reviewed.

Open access This is an open access article distributed in accordance with the Creative Commons Attribution Non Commercial (CC BY-NC 4.0) license, which permits others to distribute, remix, adapt, build upon this work non-commercially, and license their derivative works on different terms, provided the original work is properly cited, appropriate credit is given, any changes made indicated, and the use is non-commercial. See: http://creativecommons.org/licenses/by-nc/4.0/.

ORCID iDs

Michel Sebastiaan Smit http://orcid.org/0000-0003-4231-3972

Hein Raat http://orcid.org/0000-0002-6000-7445

Famke Mölenberg http://orcid.org/0000-0002-5305-9730

Wilma Jansen http://orcid.org/0000-0002-4453-9054

\section{REFERENCES}

$1 \mathrm{Ng} \mathrm{M}$, Fleming T, Robinson M, et al. Global, regional, and national prevalence of overweight and obesity in children and adults during 1980-2013: a systematic analysis for the global burden of disease study 2013. Lancet 2014;384:766-81.

2 NCD Risk Factor Collaboration (NCD-RisC). Worldwide trends in body-mass index, underweight, overweight, and obesity from 1975 to 2016: a pooled analysis of 2416 population-based measurement studies in 128.9 million children, adolescents, and adults. Lancet 2017;390:2627-42.

3 Spinelli A, Buoncristiano M, Kovacs VA, et al. Prevalence of severe obesity among primary school children in 21 European countries. Obes Facts 2019;12:244-58.

4 Childhood Obesity Surveillance Initiative (COSI). Highlights 2015-17 [Factsheet], 2018.
5 WHO European Childhood Obesity Surveillance Inititive. Overweight and obesity among 6-9 year old children - Report of the third round of data collection 2012-2013, 2018.

6 Garrido-Miguel M, Cavero-Redondo I, Álvarez-Bueno C, et al. Prevalence and trends of overweight and obesity in European children from 1999 to 2016: a systematic review and meta-analysis. JAMA Pediatr 2019;173:e192430.

7 Central Bureau Statistics Netherlands. Lifestyle and (preventive) health research; demographic characteristics, 2020. Available: https://opendata.cbs.nl/statline/\#/CBS/nl/dataset/83021NED/table? ts $=1522312658353$

8 Kelly AS, Barlow SE, Rao G, et al. Severe obesity in children and adolescents: identification, associated health risks, and treatment approaches: a scientific statement from the American heart association. Circulation 2013;128:1689-712.

9 Sanders RH, Han A, Baker JS, et al. Childhood obesity and its physical and psychological co-morbidities: a systematic review of Australian children and adolescents. Eur J Pediatr 2015;174:715-46.

10 World Health Organization. Consideration of the evidence on childhood obesity for the Commission on ending childhood obesity: report of the $A D$ Hoc Working group on science and evidence for ending childhood obesity. Geneva, Switzerland, 2016.

11 Park MH, Falconer C, Viner RM, et al. The impact of childhood obesity on morbidity and mortality in adulthood: a systematic review. Obes Rev 2012;13:985-1000.

12 Pizzi MA, Vroman K. Childhood obesity: effects on children's participation, mental health, and psychosocial development. Occup Ther Health Care 2013;27:99-112.

13 Van Name M, Santoro N. Type 2 diabetes mellitus in pediatrics: a new challenge. World J Pediatr 2013;9:293-9.

14 Simmonds M, Llewellyn A, Owen CG, et al. Predicting adult obesity from childhood obesity: a systematic review and meta-analysis. Obes Rev 2016;17:95-107.

15 Ryder JR, Jacobs DR, Sinaiko AR, et al. Longitudinal changes in weight status from childhood and adolescence to adulthood. $J$ Pediatr 2019;214:187-92.

16 Withrow D, Alter DA. The economic burden of obesity worldwide: a systematic review of the direct costs of obesity. Obes Rev 2011;12:131-41.

17 Bleich SN, Vercammen KA, Zatz LY, et al. Interventions to prevent global childhood overweight and obesity: a systematic review. Lancet Diabetes Endocrinol 2018;6:332-46.

18 Poorolajal J, Sahraei F, Mohamdadi Y, et al. Behavioral factors influencing childhood obesity: a systematic review and metaanalysis. Obes Res Clin Pract 2020;14:109-18.

19 Brug J, van Stralen MM, Chinapaw MJM, et al. Differences in weight status and energy-balance related behaviours according to ethnic background among adolescents in seven countries in Europe: the ENERGY-project. Pediatr Obes 2012;7:399-411.

20 Brown T, Moore TH, Hooper L, et al. Interventions for preventing obesity in children. Cochrane Database Syst Rev 2019;7:CD001871.

21 Verjans-Janssen SRB, Gerards SMPL, Kremers SPJ, et al. Effects of the KEIGAAF intervention on the BMI z-score and energy balancerelated behaviors of primary school-aged children. Int J Behav Nutr Phys Act 2020;17:105.

22 Corder K, Schiff A, Kesten JM, et al. Development of a universal approach to increase physical activity among adolescents: the GoActive intervention. BMJ Open 2015;5:e008610.

23 Liu Z, Xu H-M, Wen L-M, et al. A systematic review and metaanalysis of the overall effects of school-based obesity prevention interventions and effect differences by intervention components. Int $J$ Behav Nutr Phys Act 2019;16:95.

24 Waters E, de Silva-Sanigorski A, Hall BJ, et al. Interventions for preventing obesity in children. Cochrane Database Syst Rev 2011:CD001871.

25 Jones RA, Sinn N, Campbell KJ, et al. The importance of long-term follow-up in child and adolescent obesity prevention interventions. Int J Pediatr Obes 2011;6:178-81.

26 McPherson A, Mackay L, Kunkel J, et al. Physical activity, cognition and academic performance: an analysis of mediating and confounding relationships in primary school children. BMC Public Health 2018;18:936.

27 Mclsaac J-LD, Kirk SFL, Kuhle S. The association between health behaviours and academic performance in Canadian elementary school students: a cross-sectional study. Int J Environ Res Public Health 2015;12:14857-71.

28 Biddle SJH, Asare M. Physical activity and mental health in children and adolescents: a review of reviews. Br J Sports Med 2011;45:886-95.

29 Biddle S, Mutrie N, Gorely T. Psychology of physical activity. 3 ed. Abingdon: Routlegde, 2015. 
30 Landry BW, Driscoll SW. Physical activity in children and adolescents. Pm R 2012;4:826-32.

31 Jansen W, Raat H, Zwanenburg EJ-van, et al. A school-based intervention to reduce overweight and inactivity in children aged 6-12 years: study design of a randomized controlled trial. BMC Public Health 2008;8:257.

32 Jansen W, Borsboom G, Meima A, et al. Effectiveness of a primary school-based intervention to reduce overweight. Int J Pediatr Obes 2011;6:e70-7.

33 Lekker fit! basisonderwijs: Gemeente Rotterdam. Available: http:// rotterdamlekkerfit.nl/doelgroepen/lekker-fit-basisonderwijs

34 Tapp A, Marketing S. Social marketing and public health: theory and practice. J Public Health 2011;33:634-34.

35 Ajzen I, Madden TJ. Prediction of goal-directed behavior: attitudes, intentions, and perceived behavioral control. J Exp Soc Psychol 1986;22:453-74.

36 Godin G, Kok G. The theory of planned behavior: a review of its applications to health-related behaviors. Am J Health Promot 1996;11:87-98.

37 Jansen W, Hazebroek-Kampschreur AA. Differences in height and weight between children living in neighbourhoods of different socioeconomic status. Acta Paediatr 1997;86:224-5.

38 Iguacel I, Michels N, Ahrens W, et al. Prospective associations between socioeconomically disadvantaged groups and metabolic syndrome risk in European children. results from the IDEFICS study. Int J Cardiol 2018;272:333-40.

39 Kember D. To control or not to control: the question of whether experimental designs are appropriate for evaluating teaching innovations in higher education. Assessment \& Evaluation in Higher Education 2003;28:89-101.

40 Leatherdale ST. Natural experiment methodology for research: a review of how different methods can support real-world research. Int $J$ Soc Res Methodol 2019;22:19-35.

41 Biklen SK, Bogdan R. On your own with naturalistic evaluation. New Directions for Program Evaluation 1986;1986:93-101.

42 Chan A-W, Tetzlaff JM, Gøtzsche PC, et al. Spirit 2013 explanation and elaboration: guidance for protocols of clinical trials. BMJ 2013;346:e7586.

43 World Health Organization (WHO). Growth reference 5-19 years. Available: https://www.who.int/growthref/en/

44 Butte NF, Garza C, de Onis M. Evaluation of the feasibility of international growth standards for school-aged children and adolescents. J Nutr 2007;137:153-7.

45 de Onis M, Lobstein T. Defining obesity risk status in the general childhood population: which cut-offs should we use? Int J Pediatr Obes 2010;5:458-60.

46 Léger LA, Lambert J. A maximal multistage 20-m shuttle run test to predict VO2 max. Eur J Appl Physiol Occup Physiol 1982;49:1-12.
47 Tomkinson GR, Lang JJ, Blanchard J, et al. The 20-m shuttle run: assessment and interpretation of data in relation to youth aerobic fitness and health. Pediatr Exerc Sci 2019;31:152-63.

48 Wendel-Vos GCW, Schuit AJ, Saris WHM, et al. Reproducibility and relative validity of the short questionnaire to assess health-enhancing physical activity. J Clin Epidemiol 2003;56:1163-9.

49 Campbell N, Gaston A, Gray C, et al. The short questionnaire to assess health-enhancing (squash) physical activity in adolescents: a validation using doubly labeled water. J Phys Act Health 2016;13:154-8

50 Goodman R. The strengths and difficulties questionnaire: a research note. J Child Psychol Psychiatry 1997;38:581-6.

51 Goedhart A, Treffers F, Widenfelt B. Vragen naar psychische problemen bij kinderen en adolescenten. Maandblad Geestelijke Volksgezondheid 2003;58:1018-35.

52 Ajzen I. Constructing a theory of planned behavior questionnaire, 2006.

53 Kenniscentrum Sport. Infographic beweegrichtlijnen, 2019.

54 Voedingscentrum. Schijf van Vijf-vak: groente en fruit.

55 Felder-Puig R, Griebler R, Samdal O, et al. Does the school performance variable used in the International health behavior in school-aged children (HBSC) study reflect students' school grades? $J$ Sch Health 2012;82:404-9.

56 Petersen AC, Crockett L, Richards M, et al. A self-report measure of pubertal status: reliability, validity, and initial norms. J Youth Adolesc 1988;17:117-33.

57 Janssens KAM, Rosmalen JGM, Ormel J, et al. Pubertal status predicts back pain, overtiredness, and dizziness in American and Dutch adolescents. Pediatrics 2011;128:553-9.

58 Borm GF, Fransen J, Lemmens WAJG. A simple sample size formula for analysis of covariance in randomized clinical trials. J Clin Epidemiol 2007;60:1234-8.

59 Gray HL, Burgermaster M, Tipton E, et al. Intraclass correlation coefficients for obesity indicators and energy Balance-Related behaviors among New York City public elementary schools. Health Educ Behav 2016;43:172-81.

60 van de Gaar VM, Jansen W, van Grieken A, et al. Effects of an intervention aimed at reducing the intake of sugar-sweetened beverages in primary school children: a controlled trial. Int J Behav Nutr Phys Act 2014;11:98.

61 Weihrauch-Blüher S, Kromeyer-Hauschild K, Graf C, et al. Current guidelines for obesity prevention in childhood and adolescence. Obes Facts 2018;11:263-76.

62 Hariton E, Locascio JJ. Randomised controlled trials - the gold standard for effectiveness research: Study design: randomised controlled trials. BJOG 2018;125:1716.

63 Rosenbaum PR, Rubin DB. The central role of the propensity score in observational studies for causal effects. Biometrika 1983;70:41-55. 